\title{
Teste de comprimento de plântula na avaliação da qualidade fisiológica de sementes de Amburana cearensis (Allemão) A.C. Smith
}

\section{Seedling length test in the evaluation of the physiological quality of Amburana cearensis (Allemão) A.C. Smith seeds}

\author{
Roberta Sales Guedes ${ }^{1 *}$; Edna Ursulino Alves ${ }^{2}$; \\ Sueli da Silva Santos-Moura ${ }^{3}$; Evio Alves Galindo ${ }^{3}$
}

\begin{abstract}
Resumo
Os testes de vigor são empregados no controle interno de qualidade pelas empresas produtoras de sementes. $\mathrm{O}$ teste de comprimento de plântulas tem potencial para fornecer informações complementares às obtidas no teste de germinação e que possibilitem estimar o potencial de emergência de plântulas em campo. Assim, o trabalho objetivou verificar a possibilidade de utilização do teste de comprimento de plântulas como um método de vigor para classificar lotes de sementes de Amburana cearensis (Allemão) A.C. Smith de acordo com a sua qualidade fisiológica. Para tanto a qualidade fisiológica de nove lotes de sementes de $A$. cearensis foi avaliada por testes de viabilidade e vigor, realizados em laboratório e campo. Empregou-se o delineamento inteiramente ao acaso e as médias foram comparadas pelo teste de Scott-Knott a 5\% de probabilidade. As sementes procedentes de Serra Negra - RN (lotes $\mathrm{L}_{1}, \mathrm{~L}_{2}$ e $\mathrm{L}_{3}$ ) atingem maior porcentagem de emergência e comprimento de plântulas, possibilitando a classificação como lotes de alto vigor. A separação mais ampla dos lotes em níveis de vigor foi obtida com os testes de comprimento de plântulas, seguido do teste de primeira contagem de germinação e de emergência em campo.
\end{abstract}

Palavras-chave: Vigor, sementes florestais, germinação, emergência

\begin{abstract}
Vigor tests are used in the internal quality control by seed producing companies. Amongst the vigor tests, the test of length of seedlings has potential to give complementary informations to the germination test and makes possible to estimate the potential of emergence of seedlings in the field. The objective of this study was to verify the possibility of using the seedling length test as a vigor method to classify soybean seed lots in agreement with their physiological quality. The physiological quality of nine $A$. cearensis seeds lots was evaluated by viability and vigor test carried out in the laboratory and field. A completely randomized design was used. The seeds coming from Serra Negra - RN (lots $\mathrm{L}_{1}, \mathrm{~L}_{2}$ and $\mathrm{L}_{3}$ ) reached higher percentage of emergence and seedling length, allowing the classification as lots of high vigor. The averages were compared by the Scott-Knott test at 5\%. Wider separation of lots in vigor levels was obtained in tests of seedling length, followed by the test first germination and field emergence.
\end{abstract}

Key words: Vigor, forest seeds, germination, emergence

\footnotetext{
Bióloga, Prof $^{\mathrm{a}} \mathrm{Dr}^{\mathrm{a}}$, Dept ${ }^{\mathrm{o}}$ de Fitotecnia, Centro de Ciências Agrárias, Universidade Federal de Santa Catarina, UFSC, Florianópolis, SC. E-mail: guedes.r.s@ufsc.br

2 Eng $^{\mathrm{a}} \mathrm{Agr}^{\mathrm{a}}$, Prof $^{\mathrm{a}} \mathrm{Dr}^{\mathrm{a}}$, Dept ${ }^{\mathrm{o}}$ de Fitotecnia e Ciências Ambientais, Universidade Federal da Paraíba, UFPB, Centro de Ciências Agrárias, CCA, Areia, PB. E-mail: ednaaursulino@cca.ufpb.br

3 Discentes de Doutorado em Agronomia, UFPB, CCA, Areia, PB. E-mail: sssantosagro@hotmail.com; eviogalindoea@gmail. com

* Autor para correspondência
} 


\section{Introdução}

Amburana cearensis (Allemão) A.C. Smith é uma árvore frondosa, típica da caatinga, pertencente à família Fabaceae conhecida popularmente de cumaru, amburana-de-cheiro ou cerejeira (CANUTO et al., 2008). A árvore atinge 10 a 12 $\mathrm{m}$ de altura, possui flores brancas, vagem achatada e escura, assim como casca aromática com odor peculiar de cumarina (MAIA, 2004). Do ponto de vista econômico, a espécie é de valiosa importância comercial, dada as suas várias aplicações, sendo largamente empregada na perfumaria (CANUTO; SILVEIRA; BEZERRA, 2010), na carpintaria em geral (CANUTO et al., 2008) devido à sua reconhecida durabilidade. Além disso é empregada na medicina popular, sendo a casca da árvore e as sementes utilizadas na produção de medicamentos populares destinados ao tratamento de afecções pulmonares, tosses, asma, bronquite e coqueluche (LORENZI; MATOS, 2002).

A avaliação da qualidade fisiológica é um importante componente nos programas de controle de qualidade destinados a garantir um desempenho satisfatório das sementes e a estimativa desta qualidade é realizada rotineiramente aplicandose o teste de germinação, o qual é conduzido em condições ideais (MUNIZ et al., 2004). Entretanto, Byrum e Copeland (1995) questionam a validação deste teste para prognosticar o comportamento das sementes no campo, uma vez que as condições para germinação em campo nem sempre são favoráveis, estando as sementes sujeitas às situações adversas, de forma que a porcentagem de emergência de plântulas no campo é geralmente menor que a obtida no teste de germinação.

A utilização do teste de germinação, portanto, como único critério de avaliação do potencial de desempenho das sementes gera insatisfação e insegurança por parte dos produtores e consumidores de sementes, embora a metodologia do teste de germinação tenha sido aperfeiçoada nos últimos 30 anos (McDONALD, 2002). Face a isto, novos procedimentos de testes em sementes foram formulados, os quais são conhecidos como testes de vigor de sementes, que disponibilizam as medidas mais sensíveis da qualidade fisiológica das sementes e permitem estimar, com relativa precisão, o potencial de desempenho de lotes de sementes.

Dentre os diferentes métodos utilizados para avaliar o vigor das sementes existem os testes que se baseiam no desempenho das plântulas, realizados em laboratório, em condições controladas ou de campo. Desta forma, junto ao teste de germinação podem ser avaliadas certas características da germinação ou das plântulas, consideradas como expressão do vigor (ARTHUR; TONKING, 1991). $\mathrm{Na}$ maioria das pesquisas desenvolvidas no Brasil os principais testes que avaliam o desempenho das plântulas são a primeira contagem de germinação, o índice de velocidade de germinação, o comprimento da plântula e massa seca de plântulas.

A primeira contagem da germinação avalia a porcentagem de plântulas normais que são obtidas por ocasião da primeira contagem do teste de germinação na amostra em análise, sendo eficiente para determinar o vigor das sementes (NAKAGAWA, 1999). Um dos conceitos mais antigos de vigor de sementes está relacionado à velocidade de germinação (AOSA, 1983). Embora os lotes de sementes estejam com porcentagens de germinação semelhantes, frequentemente registramse diferenças na velocidade de germinação, sugerindo que existem diferenças de vigor entre eles (NAKAGAWA, 1999), sendo mais vigorosas, portanto aquelas sementes com maior velocidade de germinação.

A análise do crescimento de plântulas pode ser mensurada por meio de duas grandezas físicas, o comprimento e a massa seca (VANZOLINI et al., 2007), sendo que ambas impossibilitam a subjetividade do analista na inferência dos resultados. A determinação do comprimento médio das plântulas normais é realizada, tendo em vista que as amostras que expressam os maiores valores 
são mais vigorosas (NAKAGAWA, 1999) e isso decorre da maior translocação das reservas dos tecidos de armazenamento para o crescimento do eixo embrionário (DAN et al., 1987). Os testes que avaliam o comprimento de plântulas são recomendados pela Association of Official Seed Analysts (AOSA, 1983) e International Seed Testing Association (ISTA, 1981).

Mesmo havendo recomendação deste teste para avaliar o vigor de sementes, o mesmo se restringe a sementes de espécies cultivadas, com escassos relatos de usos para espécies florestais, tais como a Erytrina velutiva (GUEDES et al., 2009) e Amburana cearensis (GUEDES et al., 2013).

Deste modo, realizou-se a pesquisa com o objetivo de averiguar a possibilidade de utilização do teste de comprimento de plântulas como um método de vigor para classificar lotes de sementes de Amburana cearensis.

\section{Material e Métodos}

A pesquisa foi conduzida no Laboratório de Análise de Sementes (LAS), do Centro de Ciências Agrárias, da Universidade Federal da Paraíba, em Areia - PB. Após vibração dos ramos das árvores, as sementes de Amburana cearensis foram coletadas manualmente no chão de nove árvores matrizes, em localidades distintas, como observado na Tabela 1. Cada localidade corresponde a um lote de sementes e, após coletadas as sementes foram encaminhadas ao LAS para serem beneficiadas (retirar as alas) e em seguida realizou-se o experimento (cerca de 15 dias após a coleta).

Tabela 1. Lotes de sementes de Amburana cearensis oriundas de diferentes procedências. Areia - PB. 2012.

\begin{tabular}{|c|c|c|}
\hline Lotes & Municípios de procedência & Coordenada geográfica \\
\hline $\mathrm{L}_{1}$ & Serra Negra - RN & S $06^{\circ} 40^{\prime} 13,9^{\prime \prime} / \mathrm{W} 37^{\circ} 25^{\prime} 55.8^{\prime \prime}$ \\
\hline $\mathrm{L}_{2}$ & Serra Negra - RN & $\mathrm{S} 06^{\circ} 39^{\prime} 08.2^{\prime \prime} / \mathrm{W} 37^{\circ} 29^{\prime} 2.2^{\prime \prime}$ \\
\hline $\mathrm{L}_{3}^{2}$ & Serra Negra - RN & $\mathrm{S} 06^{\circ} 39^{\prime} 28.5^{\prime \prime} / \mathrm{W} 37^{\circ} 26^{\prime} 45.8^{\prime \prime}$ \\
\hline $\mathrm{L}_{4}$ & Santa Gertrudes - PB & S $06^{\circ} 56^{\prime} 34.8^{\prime \prime} / \mathrm{W} 37^{\circ} 24^{\prime} 01.5^{\prime \prime}$ \\
\hline $\mathrm{L}_{5}^{4}$ & São Bentinho - PB & $\mathrm{S} 06^{\circ} 51^{\prime} 48.2^{\prime \prime} / \mathrm{W} 37^{\circ} 44^{\prime} 07.6^{\prime \prime}$ \\
\hline $\mathrm{L}_{6}$ & Patos - PB & S $06^{\circ} 58^{\prime} 55.9^{\prime \prime} / \mathrm{W} 37^{\circ} 19^{\prime} 50.4^{\prime \prime}$ \\
\hline $\mathrm{L}_{7}^{0}$ & Malta - PB & $\mathrm{S} 06^{\circ} 55^{\prime} 00.1^{\prime \prime} / \mathrm{W} 37^{\circ} 28^{\prime} 46.7^{\prime \prime}$ \\
\hline $\mathrm{L}_{8}$ & Aparecida $-\mathrm{PB}$ & S $06^{\circ} 54^{\prime} 40.9^{\prime \prime} / \mathrm{W} 37^{\circ} 49^{\prime} 77.6^{\prime \prime}$ \\
\hline $\mathrm{L}_{9}^{\circ}$ & Souza - PB & $\mathrm{S} 06^{\circ} 53^{\prime} 58.2^{\prime \prime} / \mathrm{W} 37^{\circ} 24^{\prime} 17.5^{\prime \prime}$ \\
\hline
\end{tabular}

Fonte: Elaboração dos autores.

A qualidade fisiológica das sementes foi avaliada com os seguintes testes:

O teste de germinação foi conduzido com quatro repetições de 25 sementes para cada lote, sendo previamente tratadas com fungicida $\operatorname{Captan}^{\circledR}$, na proporção de $240 \mathrm{~g}$ para $10.000 \mathrm{~g}$ de sementes. Para condução do teste utilizou-se o substrato vermiculita e a temperatura de $35^{\circ} \mathrm{C}$ (GUEDES et al., 2010b), sendo o substrato umedecido com água destilada a $60 \%$ de sua capacidade de retenção e as sementes foram semeadas a uma profundidade de $3,5 \mathrm{~cm}$
(GUEDES et al., 2010a), em bandejas plásticas com dimensões de $0,40 \times 0,40 \times 0,11 \mathrm{~m}$ e fotoperíodo de 12 h. A avaliação da porcentagem final de germinação foi efetuada aos 12 dias considerandose como sementes germinadas aquelas que haviam emitido o epicótilo acima do substrato (plântulas normais) (GUEDES et al., 2010b).

A primeira contagem de geminação foi conduzida conjuntamente com o teste de germinação, computando-se o número de sementes germinadas aos oito dias após a instalação do teste (GUEDES 
et al., 2010b), sendo os dados expressos em percentagem. O índice de velocidade de germinação (IVG) foi avaliado conjuntamente com o teste de germinação, com a realização de contagens diárias das sementes germinadas, do $8^{\circ}$ até o $12^{\circ}$ dia após a semeadura (GUEDES et al., 2010b) de acordo com a fórmula proposta por Maguire (1962).

Para a emergência em campo utilizaram-se quatro repetições de 25 sementes de cada lote, as quais foram semeadas em sulcos de 1,0 m de comprimento e distanciadas $20 \mathrm{~cm}$ entre si, a uma profundidade de $3,5 \mathrm{~cm}$, conforme recomendação de Guedes et al. (2010a). Durante o período de condução do teste a temperatura média do ambiente foi de $35{ }^{\circ} \mathrm{C}$ e umidade relativa do ar de $67 \%$. O umedecimento dos canteiros foi diário e as contagens do número de plântulas emergidas efetuadas diariamente dos 12 até aos 18 dias após a semeadura (GUEDES et al., 2010a), considerandose como emergidas as plântulas que haviam emitido o epicótilo acima do substrato, sendo os resultados expressos em porcentagem.

Para avaliação do comprimento de plântulas as sementes foram submetidas aos procedimentos adaptados da AOSA (1983) e descritos por Nakagawa (1999), uma linha foi traçada no terço superior do papel toalha (germitest) no sentido longitudinal, o qual foi umedecido previamente com água destilada na quantidade equivalente a três vezes a massa seca do papel. Quatro repetições de 20 sementes de $A$. cearensis foram posicionadas de forma que a micrópila estivesse voltada para a parte inferior do papel, os rolos foram acondicionados em sacos plásticos posicionados verticalmente no germinador por sete dias a $25{ }^{\circ} \mathrm{C}$ e ausência de luz. Ao final deste período foi efetuada a medida da raiz primária das plântulas normais utilizando-se uma régua, sendo os resultados médios por plântulas expressos em centímetros. Também no final do teste de germinação e de emergência em campo, a raiz primária e a parte aérea das plântulas normais de cada repetição foram medidas com o auxílio de uma régua graduada em centímetros, sendo os resultados expressos em cm plântula ${ }^{-1}$.

O experimento foi conduzido segundo o delineamento inteiramente ao acaso, exceto os dados de emergência em campo que foram em blocos ao acaso, utilizando-se quatro repetições por tratamento (lote). Os dados foram submetidos à análise de variância e suas médias foram comparadas pelo teste de Scott-Knott a 5\% de probabilidade, sem transformação de dados.

\section{Resultados e Discussão}

Na Tabela 2 encontram-se os dados referentes à avaliação inicial da qualidade fisiológica dos nove lotes de sementes de Amburana cearensis, os quais diferiram entre si quando se observa o percentual de germinação, entretanto não é possível emitir parecer acerca do vigor das sementes, pois conforme comentaram Santos; Paula (2005) a qualidade fisiológica da semente pode ser avaliada por meio da viabilidade e vigor. A viabilidade procura determinar se a semente encontra-se viva ou morta, enquanto que a avaliação do vigor permite detecção de diferenças na qualidade fisiológica de lotes que com poder germinativo semelhante (SANTOS; PAULA, 2005; FLAVIO; PAULA, 2010). Os maiores porcentuais de germinação foram observados nos lotes $\mathrm{L}_{1}$ e $\mathrm{L}_{3}$, com 98 e $92 \%$ de germinação, respectivamente. A avaliação das características fisiológicas das sementes é de fundamental importância para a análise da qualidade do lote das espécies florestais, podendo avaliar com eficácia o processo de emergência de plântulas, sobrevivência e desenvolvimento das mudas em campo (SANTOS et al., 2012). 
Tabela 2. Caracterização da qualidade fisiológica inicial dos lotes de sementes de Amburana cearensis pela germinação $(\mathrm{G})$, primeira contagem de germinação (PCG), índice de velocidade de germinação (IVG) e emergência em campo (EC). Areia - PB. 2012.

\begin{tabular}{ccccc}
\hline Lotes & G (\%) & PCG (\%) & IVG & EC (\%) \\
\hline $\mathrm{L}_{1}$ & $98,00 \mathrm{a}$ & $66,00 \mathrm{a}$ & $2,941 \mathrm{a}$ & $90,00 \mathrm{a}$ \\
$\mathrm{L}_{2}$ & $88,00 \mathrm{~b}$ & $33,00 \mathrm{~b}$ & $2,332 \mathrm{~b}$ & $86,00 \mathrm{~b}$ \\
$\mathrm{~L}_{3}$ & $92,00 \mathrm{a}$ & $26,00 \mathrm{c}$ & $2,572 \mathrm{~b}$ & $87,00 \mathrm{~b}$ \\
$\mathrm{~L}_{4}$ & $79,00 \mathrm{c}$ & $14,00 \mathrm{e}$ & $2,171 \mathrm{~b}$ & $72,00 \mathrm{~d}$ \\
$\mathrm{~L}_{5}$ & $86,00 \mathrm{~b}$ & $21,00 \mathrm{~d}$ & $2,384 \mathrm{~b}$ & $78,00 \mathrm{c}$ \\
$\mathrm{L}_{6}$ & $82,00 \mathrm{c}$ & $16,00 \mathrm{e}$ & $2,276 \mathrm{~b}$ & $79,00 \mathrm{c}$ \\
$\mathrm{L}_{7}$ & $78,00 \mathrm{c}$ & $9,00 \mathrm{f}$ & $2,124 \mathrm{~b}$ & $76,00 \mathrm{c}$ \\
$\mathrm{L}_{8}$ & $66,00 \mathrm{~d}$ & $2,00 \mathrm{~g}$ & $1,449 \mathrm{c}$ & $67,00 \mathrm{e}$ \\
$\mathrm{L}_{9}$ & $77,00 \mathrm{c}$ & $4,00 \mathrm{~g}$ & $1,632 \mathrm{c}$ & $70,00 \mathrm{~d}$ \\
\hline $\mathrm{CV}$ & 3,07 & 14,54 & 8,49 & 2,39 \\
\hline
\end{tabular}

Médias seguidas de mesma letra, minúscula na coluna, não diferem estatisticamente entre si, a 5\% de probabilidade pelo teste de Scott-Knott. A - $\mathrm{L}_{1}, \mathrm{~L}_{2}$ e $\mathrm{L}_{3}$ (Serra Negra - RN); B - $\mathrm{L}_{4}, \mathrm{~L}_{5}$ e $\mathrm{L}_{6}$ (Santa Gertrudes, São Bentinho e Patos - PB, respectivamente); $\mathrm{C}-\mathrm{L}_{7}, \mathrm{~L}_{8}$ e $\mathrm{L}_{9}$ (Malta, Aparecida e Souza - PB, , respectivamente).

Fonte: Elaboração dos autores.

Quanto ao vigor, avaliado pelo teste de primeira contagem de germinação, que corresponde ao porcentual de sementes germinadas no $8^{\circ}$ dia, registrou-se maior sensibilidade do teste quando comparado aos dados obtidos no teste de germinação, classificando o lote $\mathrm{L}_{1}$ (Serra Negra - RN) como de qualidade superior e os lotes procedentes de Aparecida e Souza - PB $\left(\mathrm{L}_{8}\right.$ e $\mathrm{L}_{9}$, respectivamente) como de baixa qualidade, quando comparados aos demais.

O teste de primeira contagem de germinação, pela facilidade de execução, pode ser utilizado para obtenção de informações preliminares sobre o vigor dos lotes. Este é o caso das sementes de Cucumis sativus L. em que este teste pode ser utilizado rotineiramente para se obter informações sobre o vigor de lotes (BHERING et al., 2000). Em sementes de Brassica napus L. var. oleifera, Ávila et al. (2005) verificaram que a primeira contagem da germinação correlacionou-se com a emergência das plântulas no campo, distinguindo lotes em níveis de vigor, indicando ser o teste que melhor estimou a emergência em campo. Nesse sentido, Nakagawa (1999), destacou que a primeira contagem de germinação muitas vezes expressa melhor as diferenças de velocidade de germinação entre lotes do que os índices de velocidade de germinação (IVG), uma vez que a primeira contagem traz a porcentagem de sementes que germinaram no referido teste, enquanto que o IVG se refere a uma estimativa do número médio de plântulas normais por dia. Sabe-se que a qualidade da semente é a soma de diversos atributos que contribuem para obtenção de plântulas mais vigorosas, com germinação e emergência mais rápida (CARVALHO et al., 2012). Tais resultados foram observados para o índice de velocidade de germinação das sementes de $A$. cearensis (Tabela 2), no qual as sementes do lote $\mathrm{L}_{1}$, procedentes de Serra Negra - RN foram superiores estatisticamente aos demais lotes. Por meio desse resultado é possível inferir que o lote $\mathrm{L}_{1}$ é o mais vigoroso, quando comparado com os demais lotes, o que está de acordo com os demais testes, inclusive com a emergência das plântulas em campo (Tabela 2). Esta alta velocidade evidencia o crescimento mais rápido das plântulas deste lote, $\mathrm{o}$ que pode favorecê-las em campo ou na produção de mudas, para que haja um maior aproveitamento de nutrientes e água, além de ficar por um menor período expostas às condições adversas.

Quando colocadas para germinar em condições de campo as sementes de $A$. cearensis do lote $\mathrm{L}_{1}$ 
(Serra Negra - RN) atingiram maior porcentagem de emergência (90\%), possibilitando a classificação como um lote de alta qualidade fisiológica, seguido dos lotes $\mathrm{L}_{2}$ e $\mathrm{L}_{3}$ (Serra Negra $-\mathrm{RN}$ ). Os resultados do teste de emergência de plântulas em campo também indicaram a baixa qualidade dos lotes $\mathrm{L}_{4}, \mathrm{~L}_{8}$ e $\mathrm{L}_{9}$, cujas sementes foram coletadas em Santa Gertrudes, Aparecida e Souza, na Paraíba, respectivamente.
Com base na avaliação do comprimento total das plântulas foi possível identificar os lotes $\mathrm{L}_{1}, \mathrm{~L}_{2}$ e $\mathrm{L}_{3}$, prodedentes de matrizes de Serra Negra - RN, como os de mais alto vigor porque foram registradas as mais altas taxas de crescimento. Enquanto isso os lotes $\mathrm{L}_{8}$ e $\mathrm{L}_{9}$ podem ser considerados de baixo vigor devido ao fato de desenvolverem as plântulas com os menores comprimentos (Figura 1).

Figura 1. Teste de comprimento de plântulas de Amburana cearensis oriundas de sementes de diferentes locais de coleta. $\mathrm{L}_{1}, \mathrm{~L}_{2}$ e $\mathrm{L}_{3}$ (Serra Negra/RN); $\mathrm{L}_{4}-$ Santa Gertrudes/PB, $\mathrm{L}_{5}$ São Bentinho/PB L 6 - Patos/PB, L - Malta/PB, $\mathrm{L}_{8}-$ Aparecida/PB e $\mathrm{L}_{9}-$ Souza/PB). Areia - PB. 2012.

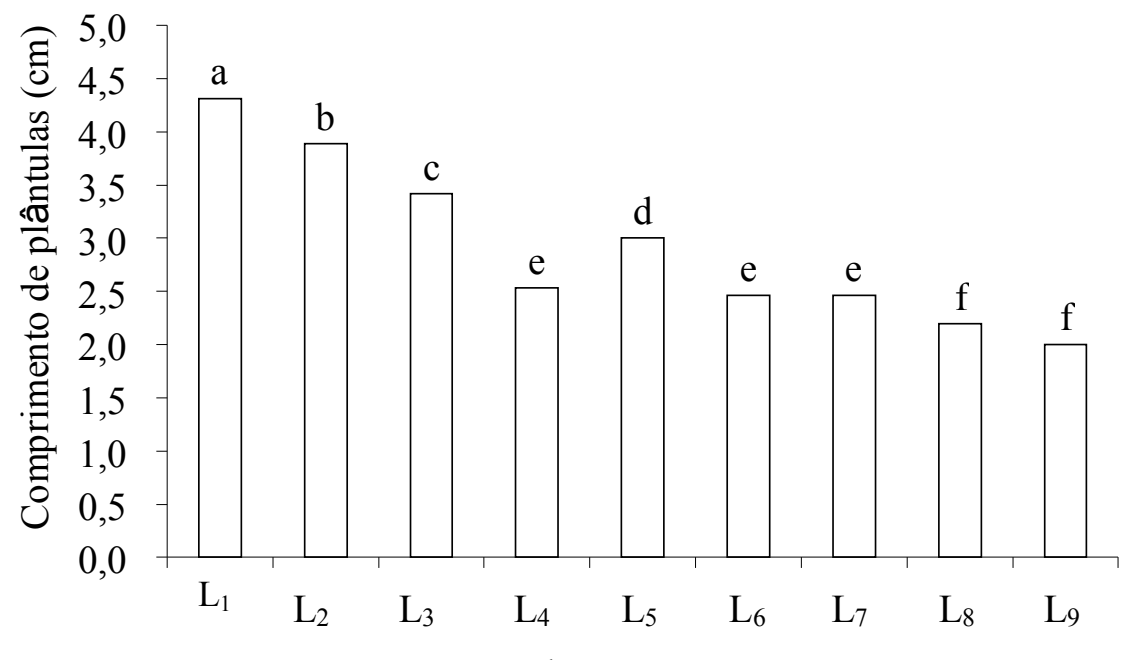

Lotes de sementes

Fonte: Elaboração dos autores.

Embora as sementes de A. cearensis tenham sido coletadas no mesmo período e mantidas nas mesmas condições de armazenamento verifica-se que há uma significativa diferença no percentual de germinação entre os lotes. Assim fica evidente que nem sempre é possível detectar as causas desta diferença de comportamento germinativo entre lotes de sementes da mesma espécie, mas acreditase que este aspecto - diferença de comportamento fisiológico dentro da mesma espécie - que ainda não está bem estudado, se deva a diferenças de condições climáticas durante as fases de maturação das sementes, às condições de secagem, à genética das matrizes ou ao ambiente em que foram obtidas. Possivelmente dentro de uma mesma espécie existam variações individuais devido às influências ambientais durante o desenvolvimento das sementes e da variabilidade genética (TURNBULL, 1975; SANTOS et al., 2009). A alta variabilidade genética devido ao estádio relativamente selvagem, sem domesticação, aliado à alogamia, têm sido apontadas como as causas da grande variação no processo germinativo de sementes de espécies florestais nativas do Brasil (SANTOS et al., 2009), o que justificaria os resultados obtidos com as sementes de $A$. cearensis quanto ao comportamento germinativo.

Esta separação dos lotes de sementes de $A$. cearensis em diferentes níveis de vigor torna-se 
fundamental, especialmente na tomada de decisão referente ao armazenamento e a produção de mudas, uma vez que a probabilidade de sucesso na produção de mudas pode aumentar invariavelmente com o uso de sementes de alto vigor. Conforme Schuch, Nedel e Assis (1999), nas sementes vigorosas há maior velocidade nos processos metabólicos, propiciando emissão mais rápida e uniforme da raiz primária no processo de germinação, maiores taxas de crescimento e produção de plântulas com maior tamanho inicial. Assim, se o propósito for a obtenção de plântulas o mais rapidamente possível para a produção de mudas, seria recomendável seguir as indicações do teste de emergência, utilizando sementes mais vigorosas $\left(\mathrm{L}_{1}, \mathrm{~L}_{2}\right.$ e $\left.\mathrm{L}_{3}\right)$, descartando-se aquelas de lotes (procedências) com valores muito abaixo da média do lote $\left(\mathrm{L}_{4}, \mathrm{~L}_{8}\right.$ e $\left.\mathrm{L}_{9}\right)$.

O teste de comprimento de plântulas visa determinar o vigor de lotes de sementes, avaliandose o comprimento médio das plântulas normais ou de uma de suas partes (raiz primária, hipocótilo, epicótilo), quando colocadas a germinar em condições controladas de ambiente, em geral idênticas às empregadas no teste de germinação (NAKAGAWA, 1999). Quando este teste foi aplicado nas sementes de $A$. cearensis verificou-se que o comprimento das plântulas foi afetado pelo vigor das sementes (Figura 1), onde as sementes de mais alto vigor (Lote $\mathrm{L}_{1}$ ) produziram plântulas com maior comprimento, enquanto que os lotes $\mathrm{L}_{8}$ e $\mathrm{L}_{9}$, compostos de sementes de baixo vigor produziram plântulas com menor comprimento. $\mathrm{O}$ alto vigor das sementes acelerou os processos metabólicos das sementes de $A$. cearensis, propiciando germinação mais rápida e uniforme. Este comportamento pode refletir também na emergência mais rápida e uniforme quando semeadas nos viveiros de produção de mudas.

Os resultados obtidos para o comprimento das plântulas de $A$. cearensis corroboram com a informação de Dan et al. (1987), o qual mencionou que as sementes vigorosas originam plântulas com maior taxa de crescimento em função da maior capacidade de transformação do suprimento de reservas dos tecidos de armazenamento e da maior incorporação destes pelo eixo embrionário. Desta forma, as amostras com maiores valores de comprimento médio de plântulas normais ou das partes destas, são consideradas mais vigorosas, como é o caso do lote $\mathrm{L}_{1}$, proveniente de Serra Negra - RN.

O que se observa com estes dados é que indiretamente a maior velocidade de germinação reflete em maiores taxas de crescimento inicial de plântulas e, isto amplia a probabilidade de sucesso durante o estabelecimento da plântula, uma vez que o crescimento rápido, tanto da raiz primária quanto da parte aérea, possibilita um aproveitamento mais rápido das reservas hídricas e nutricionais do solo, bem como melhor desenvolvimento dos processos fisiológicos. Schuch, Nedel e Assis (1999) mencionaram que se as plântulas de maior vigor emergissem rapidamente do solo e iniciassem o processo fotossintético, provavelmente manteriam essas maiores taxas de crescimento também ao longo do período de desenvolvimento da espécie, devido ao maior tamanho inicial que as de menor vigor, o que de fato é essencial para produção de mudas. Portanto, recomenda-se a medição da raiz principal das plântulas normais de $A$. cearensis como parâmetro mais confiável para avaliações do vigor. A obtenção destes dados concorda com outros autores que propuseram ser o comprimento das plântulas mais sensível para diferenciar lotes de sementes de soja (VANZOLINI et al., 2007). Os testes de comprimento de plântulas e de emergência em campo também foram indicados por Guedes et al. (2009) como viáveis para separação de lotes de sementes da espécie florestal Erythrina velutina Willd.

Deste modo a medição da raiz principal das plântulas normais de Amburana cearensis é recomendada para avaliações do vigor. 


\section{Referências}

ARTHUR, T. J.; TONKIN, J. H. B. Testando o vigor da semente. Informativo ABRATES, Londrina, v. 1, n. 3, p. 38-41, 1991.

ASSOCIATION OF OFFICIAL SEED ANALYSTS - AOSA. Seed vigor testing handbook. East Lansing: AOSA, 1983. 93 p. (Contribution, 32).

ÁVILA, M. R.; BRACCINI, A. L; SCAPIM, C. A.; MARTORELLI, D. T.; ALBRECHT, L. P. Testes de laboratório em sementes de canola e a correlação com a emergência das plântulas em campo. Revista Brasileira de Sementes, Pelotas, v. 27, n. 1, p. 62-70, 2005.

BHERING, M. C.; DIAS, D. C. F. S.; GOMES, J. M.; BARROS, D. I. Métodos para avaliação do vigor de sementes de pepino. Revista Brasileira de Sementes, Londrina, v. 22, n. 2, p. 171-175, 2000.

BYRUM, J. R.; COPELAND, L. O. Variability in vigour testing of maize (Zea mays L.) seed. Seed Science and Technology, Zürich, v. 23, n. 2, p. 543-549, 1995.

CANUTO, K. M.; SILVEIRA, E. R.; BEZERRA, A. M. E. Estudo fitoquímico de espécimes cultivados de cumaru (Amburana cearensis A. C. Smith). Química Nova, São Paulo, v. 33, n. 3, p. 662-666, 2010.

CANUTO, K. M.; SILVEIRA, E. R.; BEZERRA, A. M. E.; LEAL, L. K. A. M.; VIANA, G. S. B. Uso de plantas jovens de Amburana cearensis A. C. Smith: alternativa para preservação e exploração econômica da espécie. Petrolina: EMBRAPA, 2008. 24 p. (EMBRAPA SemiÁrido. Documentos, 208).

CARVALHO, T. C.; GRZYBOWSKI, C. R. S.; OHLSON, O. C.; PANOBIANCO, M. Comparação da qualidade fisiológica de sementes de soja convencional e de sua derivada transgênica. Revista Brasileira de Sementes, Lavras, v. 34, n. 1, p. 164-170, 2012.

DAN, E. L.; MELLO, V. D. C.;WETZEL, C. T.; POPINIGIS, F.; ZONTA, E. P. Transferência de matéria seca como método de avaliação de vigor de sementes de soja. Revista Brasileira de Sementes, Brasília, v. 9, n. 2, p. 45-55, 1987.

FLAVIO, J. J. P.; PAULA, R. C. Testes de envelhecimento acelerado e de condutividade elétrica em sementes de Dictyoloma vandellianum A. Juss. Scientia Forestalis, Piracicaba, v. 38, n. 87, p. 391-399, 2010.

GUEDES, R. S.; ALVES, E. U.; GONÇALVES, E. P.; VIANA, J. S.; BRUNO, R. L. A.; COLARES, P. N. Q. Resposta fisiológica de sementes de Erythrina velutina Willd. ao envelhecimento acelerado. Semina. Ciências Agrárias, Londrina, v. 32, n. 2, p. 323-330, 2009.
GUEDES, R. S.; ALVES, E. U.; GONÇALVES, E. P.; BRAGA JÚNIOR, J. M.; VIANA, J. S.; COLARES, P. N. Q. Substratos e temperaturas para testes de germinação e vigor de sementes de Amburana cearensis (Allemão) A.C. Smith. Revista Árvore, São Paulo, v. 34, n. 1, p. $57-$ 64, 2010a.

GUEDES, R. S.; ALVES, E. U.; GONÇALVES, E. P.; VIANA, J. S.; FRANÇA, P. R. C.; LIMA, C. R. Umedecimento do substrato e temperatura na germinação e vigor de sementes de Amburana cearensis (All.) A.C. Smith. Revista Brasileira de Sementes, Lavras, v. 32, n. 3, p. 116-122, 2010 b.

GUEDES, R. S.; ALVES, E. U.; COSTA, E. M. T.; SANTOS-MOURA, S. S.; SILVA, R. S.; CRUZ, F. R. S. Avaliação do potencial fisiológico de sementes de Amburana cearensis (Allemão) A.C. Smith. Bioscience Journal, Uberlândia, v. 29, n. 4, p. 859-866, 2013.

INTERNATIONAL SEED TESTING ASSOCIATION ISTA. Handbook of vigour test methods. Zurich, 1981. $70 \mathrm{p}$.

LORENZI, H.; MATOS, F. J. A. Plantas medicinais no Brasil: nativas e exóticas. São Paulo: Instituto Plantarum de Estudos da Flora Ltda., 2002. 512 p.

MAGUIRE, J. D. Speed of germination-aid in selection and evaluation for seedlig emergence and vigor. Crop Science, Madson, v. 2, n. 1, p. 176-177,1962.

MAIA, G. N. Caatinga: árvores e arbustos e suas utilidades. São Paulo: D \& Z Ed, 2004. 413 p.

McDONALD, M. B. Standardization of seed vigour tests. In: INTERNATIONAL SEED SEMINAR: TRADE PRODUCTION AND TECHNOLOGY, 2002, Santiago. Proceedings... Santiago: E-Library, 2002. p. 200-208.

MUNIZ, M. F. B.; GONÇALVES, N.; GARCIA, D. C.; KULCZYNSKI, S. M. Comparação entre métodos para avaliação da qualidade fisiológica e sanitária de sementes de melão. Revista Brasileira de Sementes, Pelotas, v. 26, n. 2, p. 144-149, 2004.

NAKAGAWA, J. Testes de vigor baseados no desempenho das plântulas. In: KRZYZANOSWKI, F. C.; VIEIRA, R. D.; FRANÇA NETO, J. B. (Ed.). Vigor de sementes: conceitos e testes. Londrina: ABRATES, 1999. p. 21-2.24.

SANTOS, S. R. G.; PAULA, R. C. Teste de condutividade elétrica para avaliação da qualidade fisiológica de sementes de Sebastiania commersoniana (Bail.) Smith \& Downs - Euphorbiaceae. Revista Brasileira de Sementes, Londrina, v. 27, n. 2, p. 136-145, 2005. 
SANTOS, F. S.; PAULA, R. C.; SABONARO, D. Z.; VALADARES, J. Biometria e qualidade fisiológica de sementes de diferentes matrizes de Tabebuia chrysotricha (Mart. ex A. DC.) Standl. Scientia Forestalis, Piracicaba, v. 37, n. 82, p. 163-173, 2009.

SANTOS, P. L.; FERREIRA, R. A.; ARAGÃO, A. G.; AMARAL, L. A.; OLIVEIRA, A. S. Estabelecimento de espécies florestais nativas por meio de semeadura direta para recuperação de áreas degradadas. Revista Árvore, São Paulo, v. 36, n. 2, p. 237-245, 2012.

SCHUCH, L. O. B.; NEDEL, J. L.; ASSIS, F. N. Crescimento em laboratório de plântulas de aveiapreta (Avena strigosa Schreb.) em função do vigor das sementes. Revista Brasileira de Sementes, Brasília, v. 21, n. 1, p. 229-234, 1999.
TURNBULL, J. W. Seed extraction and cleaning. In: REPORT ON THE FAO/DANIDA TRAINING COURSE ON FOREST SEED COLLECTION AND HANDLING, 1975, Chiang mai. Proceedings... Rome: FAO, 1975. p. 135-151.

VANZOLINI, S.; ARAKI, C. A. S.; SILVA, A. C. T. M.; NAKAGAWA, J. Teste de comprimento de plântula na avaliação da qualidade fisiológica de sementes de soja. Revista Brasileira de Sementes, Lavras, v. 29, n. 2, p. 9096, 2007. 
